\title{
Protective effect of Impella on left ventricular function after acute broad anterior wall ST elevation myocardial infarction with cardiogenic shock: cardiaovascular magnetic resonance imaging strain analysis
}

Daisuke Fukamachi ( $\square$ fukamachi.daisuke@nihon-u.ac.jp )

Nihon University Itabashi Hospital: Nihon Daigaku Igakubu Fuzoku Itabashi Byoin https://orcid.org/0000-0002-9647-3077

\section{Akimasa Yamada}

Nihon University Itabashi Hospital: Nihon Daigaku Igakubu Fuzoku Itabashi Byoin

Akihito Ohgaku

Nihon University Itabashi Hospital: Nihon Daigaku Igakubu Fuzoku Itabashi Byoin

Yutaka Koyama

Nihon University Itabashi Hospital: Nihon Daigaku Igakubu Fuzoku Itabashi Byoin Hidesato Fujito

Nihon University Itabashi Hospital: Nihon Daigaku Igakubu Fuzoku Itabashi Byoin Riku Arai

Nihon University Itabashi Hospital: Nihon Daigaku Igakubu Fuzoku Itabashi Byoin Yasunari Ebuchi

Nihon University Itabashi Hospital: Nihon Daigaku Igakubu Fuzoku Itabashi Byoin

\section{Suguru Migita}

Nihon University Itabashi Hospital: Nihon Daigaku Igakubu Fuzoku Itabashi Byoin

\section{Tomoyuki Morikawa}

Nihon University Itabashi Hospital: Nihon Daigaku Igakubu Fuzoku Itabashi Byoin

\section{Masaki Monden}

Nihon University Itabashi Hospital: Nihon Daigaku Igakubu Fuzoku Itabashi Byoin

\section{Takehiro Tamaki}

Nihon University Itabashi Hospital: Nihon Daigaku Igakubu Fuzoku Itabashi Byoin

Keisuke Kojima

Nihon University Itabashi Hospital: Nihon Daigaku Igakubu Fuzoku Itabashi Byoin Naotaka Akutsu

Nihon University Itabashi Hospital: Nihon Daigaku Igakubu Fuzoku Itabashi Byoin Nobuhiro Murata 
Nihon University Itabashi Hospital: Nihon Daigaku Igakubu Fuzoku Itabashi Byoin

\section{Saito Yuki}

Nihon University Itabashi Hospital: Nihon Daigaku Igakubu Fuzoku Itabashi Byoin

\section{Daisuke Kitano}

Nihon University Itabashi Hospital: Nihon Daigaku Igakubu Fuzoku Itabashi Byoin

\section{Mitsumasa Sudo}

Nihon University Itabashi Hospital: Nihon Daigaku Igakubu Fuzoku Itabashi Byoin

\section{Yasuo Okumura}

Nihon University Itabashi Hospital: Nihon Daigaku Igakubu Fuzoku Itabashi Byoin

\section{Norio Takei}

Nihon University Itabashi Hospital: Nihon Daigaku Igakubu Fuzoku Itabashi Byoin

\section{Research article}

Keywords: anterior ST-elevation myocardial infarction, Impella, cardiovascular magnetic resonance imaging

Posted Date: March 11th, 2021

DOl: https://doi.org/10.21203/rs.3.rs-302562/v1

License: (c) (1) This work is licensed under a Creative Commons Attribution 4.0 International License. Read Full License 


\section{Abstract \\ Background}

The clinical efficacy of Impella for high-risk percutaneous coronary intervention and cardiogenic shock remains under debate. We thus sought to investigate the protective effects on the heart by the early use of Impella before percutaneous coronary intervention using cardiovascular magnetic resonance imaging (CMR).

\section{Methods}

We evaluated cardiac magnetic resonance imaging results in 22 broad anterior ST-elevation myocardial infarction cases between 2017 and 2019. A mechanical circulation system (Impella or intra-aortic balloon pump) was implanted before percutaneous coronary intervention if needed; all patients underwent cardiac magnetic resonance imaging 2 weeks later.

\section{Results}

There were 6 patients in the Impella group and 16 in the non-Impella group; no differences were found in the door-to-balloon time ( $60 \pm 17$ vs. $58 \pm 25 \mathrm{~min}, \mathrm{P}=0.58$ ), peak creatine kinase (7922 $\pm 4864 \mathrm{vs} .6950 \pm$ $4801 \mathrm{IU} / \mathrm{L}, \mathrm{P}=0.74$ ), and hospital admission days ( $28 \pm 8$ vs. $25 \pm 7$ days, $P=0.40$ ) between the two groups; however, cardiac magnetic resonance imaging-derived left ventricular end-diastolic volume was significantly smaller in the Impella group $(140.6 \pm 28 \mathrm{vs}$. $182.5 \pm 45 \mathrm{ml}, \mathrm{P}=0.004)$. Overall diastolic strain rate (SR) at the non-infarcted area in the IMPELLA group was significantly higher as compared to non IMPELLA group (longitudinal diastolic SR: $1.1 \pm 0.4$ vs $0.7 \pm 0.3 \mathrm{~S}^{-1}, \mathrm{P}=0.04$, radial diastolic SR: $-1.8 \pm 0.4$ vs $-1.1 \pm 0.4 \mathrm{~S}^{-1}, \mathrm{P}=0.004$, circumferential diastolic $S R: 1.0 \pm 0.1 \mathrm{vs} 0.6 \pm 0.2 \mathrm{~S}^{-1}, \mathrm{P}=0.001$ ).

\section{Conclusions}

Early implantation of Impella before percutaneous coronary intervention for anterior ST-elevation myocardial infarction acutely prevented enlargement of left ventricular end-diastolic volume and worsening of diastolic SR in the remote myocardium. This study provides clinical insight into understanding the usefulness of Impella to prevent future heart failure.

\section{Background}

The survival rate of acute myocardial infarction (AMI) has generally improved due to technological advancement in Japan ${ }^{1}$; however, ST-elevation myocardial infarction (STEMI) with cardiogenic shock has a high mortality rate, even after shortening the door-to-balloon time (DTBT).${ }^{2}$ Additionally, heart failure 
associated with STEMI tends to increase due to the development of left ventricular (LV) remodeling; thereafter, prognosis worsens. ${ }^{3}$

Previously, the main treatment strategy for cardiogenic shock in AMI was to maintain hemodynamics using the mechanical circulatory support of intra-aortic balloon pumping (IABP) and veno-arterial extracorporeal membrane oxygenation (VA-ECMO). Earlier studies demonstrated that IABP reduces afterload and improves coronary blood flow ${ }^{4}$; however, although IABP stabilizes hemodynamics, afterload reduction was ineffective. Thus, IABP does not improve 30-day survival in STEMI patients with cardiogenic shock. ${ }^{5}$ While VA-ECMO is a powerful technique that assists with systemic circulation, it increases LV load and afterload.

Under these circumstances, the Impella 2.5 or 5.0 (Abiomed, Danvers, MA, USA), a percutaneous LV assist device for drug-resistant cardiogenic shock, was used since 2017 in Japan; the Impella CP (Abiomed), designed to provide a higher level of support than the Impella 2.5, was available since 2019. By drawing arterial blood from the left ventricle to the aorta, it is possible to directly unload the left ventricle. Direct LV unload lowers LV end-diastolic pressure, improving blood gas oxygenation and systemic perfusion. ${ }^{6}$ Several reports have reported that use of Impella in the acute phase increases cardiac output, improving coronary blood flow. ${ }^{7,8}$ Unexpectedly, the IMPRESS in Severe Shock trial, the first randomized pilot trial to compare the efficacy and safety of the Impella CP versus IABP, did not show 3-day and 6-month mortality in the 48 patients (24 per group) ${ }^{9}$; this was possibly due to the fact that Impella increased bleeding events, worsening renal function and cerebral infarction, and causing death during coronary treatment. ${ }^{10}$ A major concern was that the Impella was placed "after" revascularization in $80 \%$ of the study patients; an earlier study demonstrated improved survival in patients who received an Impella pre-, rather than post-percutaneous coronary intervention (PCI). ${ }^{11}$ Further, over $90 \%$ of the patients in that trial had resuscitated cardiac arrest patients. Those conditions might have lessened the beneficial effects of IMPELLA. From the standpoint of this view, we hypothesized that IMPELLA would provide some beneficial effects of LV remodeling for the patients who did not experience cardiac arrest and did receive IMPELLA before PCl. For bridging the gap between the trial-based evidence and "real-world" practice, we retrospectively evaluated the detailed LV function including myocardial deformation by cardiovascular magnetic resonance imaging (CMR) in the patients with anterior STEMI in whom IMPELLA was placed just before percutaneous coronary intervention (PCI), and they were compared to those without IMPELLA.

\section{Methods}

\section{Study population}

In order to investigate the protective effects on the heart by the early use of Impella before percutaneous coronary intervention using cardiac magnetic resonance imaging, this study retrospectively enrolled 47 consecutive patients with broad anterior STEMI who were admitted to our hospital between January 2017 and June 2019. The inclusion criterion was having undergone PCI for the first anterior STEMI, followed by 
CMRI before discharge; patients with claustrophobia, a history of old myocardial infarction, or renal failure including dialysis were excluded. Anterior AMI patients with cardiopulmonary arrest and impaired consciousness were also excluded. In all patients, the culprit lesion was the proximal left anterior descending (LAD) or left main trunk. STEMI is universally defined as myocardial infarction, where STelevation refers to two or more leads. In V2-3 leads, the ST-elevation was: $\geq 2.0 \mathrm{~mm}$ for men $>40$ years, $\geq$ $2.5 \mathrm{~mm}$ for men $<40$ years, and $\geq 1.5 \mathrm{~mm}$ for women regardless of age; the ST rise was $\geq 1.0 \mathrm{~mm}$. Figure 1 shows the flowchart for the study participants. Among 47 broad anterior STEMI patients, CMRI was not performed in 25 patients, 5 died immediately after admission, 12 had chronic kidney disease or hemodialysis, and 8 were frail; the remaining 22 patients were the study participants. All patients had consented, by the opt-out method, to use their data for study purposes. The study protocol was reviewed and approved by the Ethics Committee of Nihon University Itabashi Hospital (RK-200714-10).

\section{Data collection}

Patient characteristics and follow-up data were retrospectively obtained from our hospital records. Patient background at the time of PCI for ACS was anonymized and extracted; information included age, sex, age, body mass index (BMI), smoking history, comorbidities (hypertension, diabetes, dyslipidemia, or prior coronary disease), hemodynamic variables (heart rate, systolic blood pressure, and diastolic blood pressure), laboratory tests (hemoglobin concentration, lactate, and estimated glomerular filtration rate [eGFR]), and transthoracic echocardiographic parameters (LV ejection fraction [LVEF], LV end-diastolic volume [LVEDV], and LV end-systolic volume [LVESV]) obtained at the time of the emergency CAG. Nterminal pro-brain natriuretic peptide (NT-proBNP) levels at discharge were used for analysis.

\section{Implantation of mechanical circulatory support and post-MI medical treatments}

Impella is generally used in cardiogenic shock cases for mechanical circulatory support during $\mathrm{PCl}$ of anterior STEMI; however, IABP may be used even in shock cases. The choice between Impella or IABP was made at the physicians' discretion; in all cases, both Impella and IABP were placed just before $\mathrm{PCI}$. The Impella 2.5 or CP was used, as the 5.0 model required the use of artificial blood vessels. The patient entered the coronary care unit after PCI for intensive care. The IABP and Impella were removed after the stabilized conditions from heart failure after starting a $\beta$-blocker and renin-angiotensin system inhibitor. Thereafter, they were transferred to a general ward for undergoing cardiac rehabilitation; the amount of cardioprotective agents was carefully increased. CMRI was performed before discharge from the hospital after all pressor agents, such as DOB for infusion, were tapered. The Impella and non-Impella groups were thus retrospectively compared.

\section{Cardiovascular Magnetic Resonance Imaging}

All patients underwent CMRI within 21 days; images were acquired using a 1.5-T scanner (Ingenia; Philips Healthcare, Eindhoven, Netherlands) with retrospective electrocardiographic gating and dS Torso coil. The CMRI protocol comprised standard steady-state free precession (SSFP) cine and late gadolinium 
enhancement (LGE) MRI. Standard SSFP cine images covered the entire LV using short-axis slices and 2-, 3-, and 4-chamber views with temporal resolution $<40 \mathrm{~ms}$. Endo- and epicardial LV contours were manually drawn on short-axis cine images covering the mitral valve to the apex at end-diastole and endsystole to calculate end-diastolic and end-systolic volume, stroke volume, and ejection fraction. LV mass was calculated as the sum of myocardial volumes multiplied by the specific gravity $(1.05 \mathrm{~g} / \mathrm{mL})$ of the myocardial tissue. Papillary muscles were excluded from the LV mass.

For LV strain analysis, endo- and epicardial borders were semi-automatically drawn at end-diastole in short- and long-axis cines (excluding papillary muscles from the endocardial contour) and automatically propagated to all slices throughout the cardiac cycle. Tracking was visually reviewed and manually corrected in case of inaccurate automated border tracking. Short-axis cines were tracked to derive radial and circumferential strain, while 2-, 3, and 4-chamber-view cines were tracked to derive longitudinal strain. Based on the 16-segment model, the software algorithm calculated 2D peak strains (longitudinal, radial, and circumferential) and subsequently, by averaging the according peak values of the segments. In all analyses, strain was defined as the average of peak of the global, infarcted, and non-infarcted strain curves and systolic strain rate (SR) was the average of peak of the global, infarcted, and non-infarcted SR curves in systole, and diastolic SR was the peak in diastole (Figs. 2 and 3). LGE imaging was acquired with a T1-weighted inversion recovery gradient-echo sequence $15 \mathrm{~min}$ after contrast administration (0.15 $\mathrm{mmol} / \mathrm{kg}$; Gd-BTD03A; Gadovist, Bayer Japan, Tokyo, Japan) in three long-axis slices (2-, 3-, and 4chamber) and a stack of short-axis slices completely encompassing the LV. The infarct size was quantified by the full-width at half-maximum method; this estimated an intensity threshold from the remote myocardium as the midway between the mean intensity within the remote region, and the maximal intensity within the affected tissue in short-axis. The LV myocardium was divided into infarcted and non-infarcted areas. The segmental coronary artery distribution model from the American Society of Echocardiography and the European Association of Cardiovascular Imaging guidelines ${ }^{12}$ was used; the infarcted area was defined as the proximal LAD perfusion territory. This included segments $1-2,7-8$ and 13-16; the remaining LV myocardium was defined as the non-infarcted area.

\section{Post-discharge follow-up}

Each patient was followed up at our outpatient cardiology clinic around 3, 6, 9, and 12 months.

\section{Study assessments}

CMRI at the assessment endpoint included measurements of LVEF, LVEDV, LVESV, LV mass, and infarcted size. Strain analysis involved longitudinal, radial, and circumferential peak, systolic, and diastolic SRs in the global, infarcted, and non-infarcted areas. We also ascertained clinical events including hospitalization due to heart failure, all-cause death, non-fatal myocardial infarction (MI), stroke, stent thrombosis, and major bleeding via medical records.

\section{Statistical analysis}


Values are shown as mean \pm SD, median and interquartile ranges, and number and percentages. Continuous variables between the groups were analyzed using the Student's t-test or Mann-Whitney Utest; differences in categorical variables were tested using the Fisher's exact or Chi-squared test. All analyses were performed using SPSS version 19.0 (SPSS Inc., Chicago, IL, USA); P< 0.05 was considered statistically significant.

\section{Results}

\section{Baseline characteristics between the Impella and non- Impella groups}

Among the 22 patients with anterior STEMI, an Impella was used for mechanical support in 6 patients (2.5: 5 patients; CP: 1 patient); the remaining 16 patients (non-Impella group) received an IABP (11 patients) or no mechanical circulatory support (5 patients). There were no differences in age (68 $\pm 11 \mathrm{vs.}$ $60 \pm 12$ years, $P=0.21)$, male sex ( 83 vs. $93 \%, P=0.48)$, and BMl $\left(21 \pm 1.5\right.$ vs. $\left.24 \pm 3.3 \mathrm{~kg} / \mathrm{m}^{2}, P=0.06\right)$, nor in the coronary risk factors for hypertension, dyslipidemia, diabetes, and smoking between the two groups. One patient in each group received VA-ECMO.

On admission, no difference was observed in the lactic acid level (3.2 vs. $2.3 \mathrm{mmol} / \mathrm{l}, \mathrm{P}=0.15$ ) and eGFR ( 63 vs. $72 \mathrm{ml} / \mathrm{min}, \mathrm{P}=0.32$ ) between the two groups. No difference was found between TIMI 0 flow cases of $L A D$ on angiography before $\mathrm{PCl}(67 \%$ vs. $75 \%, \mathrm{P}=0.67)$. The DTBT did not differ between the two groups ( $60 \pm 17$ vs. $58 \pm 24 \mathrm{~min}, \mathrm{P}=0.58)$. There were no differences in transthoracic echocardiographic LVEF ( $48 \pm 11$ vs. $48 \pm 7.1 \%, P=0.98)$, LVEDV on admission $(107 \pm 16$ vs. $116 \pm 33 \mathrm{ml}, P=0.38)$, and LVESV ( $52 \pm 17$ vs. $57 \pm 18 \mathrm{ml}, \mathrm{P}=0.60$ ) between the two groups. The Impella was in place for $4 \pm 1.7$ days (Table 1 ). 
Table 1

Baseline characteristics between patients with and without an Impella

\begin{tabular}{|c|c|c|c|}
\hline & $\begin{array}{l}\text { Impella } \\
(n=6)\end{array}$ & $\begin{array}{l}\text { Non-Impella } \\
(n=16)\end{array}$ & P-Value \\
\hline Age, years & $68 \pm 11$ & $60 \pm 13$ & 0.21 \\
\hline Male gender & $5(83)$ & $15(93)$ & 0.48 \\
\hline $\mathrm{BMI}\left(\mathrm{m}^{2} / \mathrm{kg}\right)$ & $21 \pm 1.6$ & $24 \pm 3.3$ & 0.06 \\
\hline \multicolumn{4}{|c|}{ Hemodynamic variables on admission } \\
\hline Heart rate (beats/min) & $83 \pm 19$ & $86 \pm 18$ & 0.72 \\
\hline Systolic blood pressure (mmHg) & $93 \pm 25$ & $133 \pm 18$ & 0.001 \\
\hline Diastolic blood pressure $(\mathrm{mmHg})$ & $76 \pm 20$ & $88 \pm 18$ & 0.18 \\
\hline Cardiogenic Shock & $6(100)$ & $5(31)$ & 0.006 \\
\hline \multicolumn{4}{|l|}{ History or comorbidities } \\
\hline Current smoking & $1(17)$ & $8(50)$ & 0.18 \\
\hline Hypertension & $5(83)$ & $11(69)$ & 0.46 \\
\hline Diabetes Mellitus & $1(17)$ & $5(31)$ & 0.46 \\
\hline Dyslipidaemia & $6(100)$ & $8(50)$ & 0.04 \\
\hline Prior coronary disease & $0(0)$ & $0(0)$ & \\
\hline \multicolumn{4}{|l|}{ Blood values on admission } \\
\hline Lactate $(\mathrm{mmol} / \mathrm{l})$ & $3.2 \pm 1.4$ & $2.4 \pm 1.0$ & 0.15 \\
\hline $\mathrm{Hb}(\mathrm{mg} / \mathrm{dl})$ & $13 \pm 1.7$ & $14 \pm 1.1$ & 0.05 \\
\hline eGFR (mg/dl) & $63 \pm 17$ & $72 \pm 20$ & 0.32 \\
\hline \multicolumn{4}{|l|}{ Blood values at discharge } \\
\hline NT-proBNP at discharge (pg/ml) & $937(422,2093)$ & $1662(809,2776)$ & 0.24 \\
\hline \multicolumn{4}{|l|}{ Support device } \\
\hline \multicolumn{4}{|c|}{ Values are shown as the number (\%), mean \pm SD or median (interquartile ranges). } \\
\hline \multicolumn{4}{|c|}{$\begin{array}{l}\text { STEMI, ST-elevation myocardial infarction; BMI, body mass index; Hb, haemoglobin; eGFR, estimated } \\
\text { glomerular filtration rate; DAPT, dual antiplatelet therapy; CPA, cardiopulmonary arrest; IABP, intra- } \\
\text { aortic balloon pumping; VA ECMO, veno-arterial extracorporeal membrane oxygenation; LVEDV, left } \\
\text { ventricular end-diastole volume; LVEF, left ventricular ejection fraction; LVESV, left ventricular end- } \\
\text { systolic volume; NT-pro BNP, n-terminal pro brain natriuretic peptide; TIMI, thrombolysis in myocardial } \\
\text { infarction; CK, creatinine kinase; DES, drug eluting stent; CMRI, cardiac magnetic resonance image; } \\
\text { N.A., not applicable }\end{array}$} \\
\hline
\end{tabular}




\begin{tabular}{|c|c|c|c|}
\hline & $\begin{array}{l}\text { Impella } \\
(n=6)\end{array}$ & $\begin{array}{l}\text { Non-Impella } \\
(n=16)\end{array}$ & P-Value \\
\hline Mechanical ventilation & $2(33)$ & $3(16)$ & 0.47 \\
\hline IABP & $0(0)$ & $11(61)$ & 0.006 \\
\hline VA ECMO & $1(17)$ & $1(6)$ & 0.48 \\
\hline Anterior STEMI & $6(100)$ & $16(100)$ & \\
\hline \multicolumn{4}{|l|}{ Infarct-related artery } \\
\hline Left main stem & $1(17)$ & $0(0)$ & \\
\hline Left anterior descending & $5(83)$ & $16(100)$ & \\
\hline Multivessel disease & $2(33)$ & $2(13)$ & 0.26 \\
\hline \multicolumn{4}{|l|}{ Stent placement } \\
\hline Drug-eluting stent & $6(100)$ & $16(100)$ & \\
\hline Number of DES stents & $1.0 \pm 0$ & $1.1 \pm 0.3$ & 0.39 \\
\hline Initial TIMI flow 0 & $4(67)$ & $12(75)$ & 0.54 \\
\hline Final TIMI flow3 & $6(100)$ & $15(94)$ & 0.72 \\
\hline Max CK (IU/L) & $7922 \pm 4864$ & $6950 \pm 4801$ & 0.68 \\
\hline Max CK MB (IU/L) & $585 \pm 351$ & $561 \pm 400$ & 0.9 \\
\hline Door to balloon time (min) & $60 \pm 17$ & $58 \pm 24$ & 0.58 \\
\hline \multicolumn{4}{|l|}{ Echo parameter on admission } \\
\hline LVEF & $48 \pm 11$ & $48 \pm 7.1$ & 0.98 \\
\hline LVEDV (ml) & $107 \pm 16$ & $116 \pm 33$ & 0.38 \\
\hline LVESV (ml) & $52 \pm 17$ & $57 \pm 18$ & 0.60 \\
\hline Duration of IMPELLA support (days) & $4.0 \pm 1.7$ & & \\
\hline Medications at discharge & & & \\
\hline
\end{tabular}

Values are shown as the number (\%), mean \pm SD or median (interquartile ranges).

STEMI, ST-elevation myocardial infarction; BMI, body mass index; $\mathrm{Hb}$, haemoglobin; eGFR, estimated glomerular filtration rate; DAPT, dual antiplatelet therapy; CPA, cardiopulmonary arrest; IABP, intraaortic balloon pumping; VA ECMO, veno-arterial extracorporeal membrane oxygenation; LVEDV, left ventricular end-diastole volume; LVEF, left ventricular ejection fraction; LVESV, left ventricular endsystolic volume; NT-pro BNP, $\mathrm{n}$-terminal pro brain natriuretic peptide; TIMI, thrombolysis in myocardial infarction; CK, creatinine kinase; DES, drug eluting stent; CMRI, cardiac magnetic resonance image; N.A., not applicable 


\begin{tabular}{|c|c|c|c|}
\hline & $\begin{array}{l}\text { Impella } \\
(n=6)\end{array}$ & $\begin{array}{l}\text { Non-Impella } \\
(n=16)\end{array}$ & P-Value \\
\hline DAPT & $6(100)$ & $16(100)$ & \\
\hline RAS inhibitor & $6(100)$ & $16(100)$ & \\
\hline Beta blocker & $6(100)$ & $15(94)$ & 0.72 \\
\hline Statin & $6(100)$ & $16(100)$ & \\
\hline Days of coronary care unit (days) & $7.8 \pm 3.9$ & $6.0 \pm 3.0$ & 0.26 \\
\hline Days of hospital admission (days) & $28 \pm 8$ & $25 \pm 7$ & 0.40 \\
\hline Days of CMRI after admission (days) & $16 \pm 7$ & $19 \pm 8$ & 0.98 \\
\hline \multicolumn{4}{|c|}{ Values are shown as the number (\%), mean \pm SD or median (interquartile ranges). } \\
\hline \multicolumn{4}{|c|}{$\begin{array}{l}\text { STEMI, ST-elevation myocardial infarction; BMI, body mass index; Hb, haemoglobin; eGFR, estimated } \\
\text { glomerular filtration rate; DAPT, dual antiplatelet therapy; CPA, cardiopulmonary arrest; IABP, intra- } \\
\text { aortic balloon pumping; VA ECMO, veno-arterial extracorporeal membrane oxygenation; LVEDV, left } \\
\text { ventricular end-diastole volume; LVEF, left ventricular ejection fraction; LVESV, left ventricular end- } \\
\text { systolic volume; NT-pro BNP, n-terminal pro brain natriuretic peptide; TIMI, thrombolysis in myocardial } \\
\text { infarction; CK, creatinine kinase; DES, drug eluting stent; CMRI, cardiac magnetic resonance image; } \\
\text { N.A., not applicable }\end{array}$} \\
\hline
\end{tabular}

\section{CMRI assessment between the Impella and non-Impella groups}

CMRI was performed $19 \pm 8$ days after admission (Impella: $16 \pm 7$ days; non-Impella: $19 \pm 8$ days, $\mathrm{P}=$ 0.98). The LVESV ( $83 \pm 27$ vs. $119 \pm 43 \mathrm{ml}, \mathrm{P}=0.06)$ and LV mass tended to be smaller ( $80 \pm 13$ vs. $95 \pm$ $23 \mathrm{~g}, \mathrm{P}=0.07$ ), the LVEF higher ( $42 \pm 9.6$ vs. $34.9 \pm 7.4 \%, P=0.07$ ), and the LVEDV significantly smaller in the Impella than non-Impella group ( $141 \pm 28$ vs. $183 \pm 45 \mathrm{~mL}, \mathrm{P}=0.004)$; no difference was observed in the infarcted size of the LV myocardial mass ( $29 \pm 15$ vs. $30 \pm 12 \%, P=0.79$; Fig. 3 ).

Representative CMR results of the two groups are shown in Fig. 2; there were no significant differences in the global peak strains (i.e., longitudinal, radial, and circumferential) and systolic SR in all LV areas (i.e., global, infarcted, and non-infarcted areas). Radial and circumferential diastolic SRs in the global and infarcted area were higher in the Impella group (global radial diastolic SR: $-1.3 \pm 0.5$ vs. $-0.7 \pm 0.4 \mathrm{~S}^{-1}, \mathrm{P}=$ 0.006 ; infarcted radial diastolic SR: $-0.9 \pm 0.6$ vs. $-0.4 \pm 0.5 \mathrm{~S}^{-1}, \mathrm{P}=0.05$; global circumferential diastolic SR: $0.8 \pm 0.2$ vs. $0.4 \pm 0.2, P=0.004$; infarcted circumferential diastolic SR: $0.6 \pm 0.3$ vs. $0.2 \pm 0.3 S^{-1}, P=$ 0.04). Additionally, all diastolic SRs in the non-infarcted area were significantly increased in the Impella group (longitudinal diastolic SR: $1.1 \pm 0.4$ vs. $0.7 \pm 0.3 \mathrm{~S}^{-1}, \mathrm{P}=0.04$; radial diastolic SR: $-1.8 \pm 0.4$ vs. -1.1 $\pm 0.4 \mathrm{~S}^{-1}, \mathrm{P}=0.004$; circumferential diastolic SR: $1.0 \pm 0.1$ vs. $0.6 \pm 0.2 \mathrm{~S}^{-1}, \mathrm{P}=0.001$; Table 2, Fig. 4). 
Table 2

CMRI strain parameters between the patients with and without Impella

\begin{tabular}{|c|c|c|c|}
\hline CMRI strain parameters & $\begin{array}{l}\text { Impella } \\
(n=6)\end{array}$ & $\begin{array}{l}\text { Non-Impella } \\
(n=16)\end{array}$ & P-Value \\
\hline \multicolumn{4}{|l|}{ LV longitudinal } \\
\hline \multicolumn{4}{|l|}{ Global } \\
\hline Peak Strain (\%) & $-9.1 \pm 2.1$ & $-8.5 \pm 2.4$ & 0.60 \\
\hline Systolic strain rate $(1 / \mathrm{s})$ & $-0.8 \pm 0.2$ & $-0.5 \pm 0.4$ & 0.12 \\
\hline Diastolic strain rate $(1 / \mathrm{s})$ & $0.6 \pm 0.2$ & $0.5 \pm 0.2$ & 0.22 \\
\hline \multicolumn{4}{|l|}{ Infarcted area } \\
\hline Peak Strain (\%) & $-6.3 \pm 2.5$ & $-6.2 \pm 3.2$ & 0.93 \\
\hline Systolic strain rate $(1 / \mathrm{s})$ & $-0.5 \pm 0.4$ & $-0.3 \pm 0.4$ & 0.42 \\
\hline Diastolic strain rate $(1 / \mathrm{s})$ & $0.2 \pm 0.3$ & $0.3 \pm 0.3$ & 0.68 \\
\hline \multicolumn{4}{|l|}{ Non-Infarcted area } \\
\hline Peak Strain (\%) & $-13 \pm 3.3$ & $-12 \pm 4.4$ & 0.56 \\
\hline Systolic strain rate $(1 / \mathrm{s})$ & $-1.1 \pm 0.6$ & $-0.7 \pm 0.7$ & 0.2 \\
\hline Diastolic strain rate $(1 / s)$ & $1.1 \pm 0.4$ & $0.7 \pm 0.3$ & 0.04 \\
\hline \multicolumn{4}{|l|}{ LV radial } \\
\hline \multicolumn{4}{|l|}{ Global } \\
\hline Peak Strain (\%) & $20 \pm 6.5$ & $16 \pm 5.6$ & 0.18 \\
\hline Systolic strain rate (1/s) & $1.1 \pm 0.3$ & $0.9 \pm 0.3$ & 0.15 \\
\hline Diastolic strain rate $(1 / \mathrm{s})$ & $-1.3 \pm 0.5$ & $-0.7 \pm 0.4$ & 0.006 \\
\hline \multicolumn{4}{|l|}{ Infarcted area } \\
\hline Peak Strain (\%) & $16 \pm 7.3$ & $13 \pm 6.0$ & 0.29 \\
\hline Systolic strain rate (1/s) & $0.9 \pm 0.4$ & $0.7 \pm 0.4$ & 0.21 \\
\hline Diastolic strain rate $(1 / s)$ & $-0.9 \pm 0.6$ & $-0.4 \pm 0.5$ & 0.05 \\
\hline \multicolumn{4}{|l|}{ Non-Infarcted area } \\
\hline Peak Strain (\%) & $24 \pm 5.6$ & $19 \pm 8.2$ & 0.22 \\
\hline
\end{tabular}

CMRI, cardiac magnetic resonance image; LV, left ventricular 


\begin{tabular}{|c|c|c|c|}
\hline CMRI strain parameters & $\begin{array}{l}\text { Impella } \\
(n=6)\end{array}$ & $\begin{array}{l}\text { Non-Impella } \\
(n=16)\end{array}$ & P-Value \\
\hline Systolic strain rate (1/s) & $1.4 \pm 0.2$ & $1.2 \pm 0.4$ & 0.28 \\
\hline Diastolic strain rate $(1 / \mathrm{s})$ & $-1.8 \pm 0.4$ & $-1.1 \pm 0.4$ & 0.004 \\
\hline \multicolumn{4}{|l|}{ LV circumferential } \\
\hline \multicolumn{4}{|l|}{ Global } \\
\hline Peak Strain (\%) & $-12 \pm 3.3$ & $-9.9 \pm 3.4$ & 0.21 \\
\hline Systolic strain rate $(1 / \mathrm{s})$ & $-0.7 \pm 0.2$ & $-0.6 \pm 0.2$ & 0.20 \\
\hline Diastolic strain rate $(1 / \mathrm{s})$ & $0.8 \pm 0.2$ & $0.4 \pm 0.2$ & 0.004 \\
\hline \multicolumn{4}{|l|}{ Infarcted area } \\
\hline Peak Strain (\%) & $-10 \pm 4.3$ & $-8.1 \pm 4.3$ & 0.40 \\
\hline Systolic strain rate (1/s) & $-0.6 \pm 0.2$ & $-0.4 \pm 0.3$ & 0.26 \\
\hline Diastolic strain rate $(1 / \mathrm{s})$ & $0.6 \pm 0.3$ & $0.2 \pm 0.3$ & 0.04 \\
\hline \multicolumn{4}{|l|}{ Non-Infarcted area } \\
\hline Peak Strain (\%) & $-15 \pm 2.2$ & $-12 \pm 4.0$ & 0.17 \\
\hline Systolic strain rate (1/s) & $-0.9 \pm 0.1$ & $-0.8 \pm 0.3$ & 0.36 \\
\hline Diastolic strain rate $(1 / \mathrm{s})$ & $1.0 \pm 0.1$ & $0.6 \pm 0.2$ & 0.001 \\
\hline
\end{tabular}

\section{Clinical outcomes}

No difference was found in the extent of infarction as evidenced by maximum creatine kinase $(7922 \pm$ 4864 vs. $6950 \pm 4801 \mathrm{IU} / \mathrm{L}, \mathrm{P}=0.74)$ and creatine kinase-MB ( $585 \pm 351$ vs. $561 \pm 400 \mathrm{IU} / \mathrm{L}, \mathrm{P}=0.93)$ between the two groups, nor in the length of hospital admission ( $28 \pm 8$ vs. $25 \pm 7$ days, $P=0.40$ ) and NTproBNP level at discharge $(937$ [422, 2093] vs. 1662 [809, 2776], $P=0.24$; Table 1$)$. In both groups, all patients were discharged on foot. During the median follow-up period of $434 \pm 302$ days, 1 (17\%) patient in the Impella group experienced a major bleeding event (gastrointestinal bleeding) 450 days after discharge; 3 (19\%) non-Impella patients were hospitalized due to heart failure.

\section{Discussion}

This study has two major findings: 1) with similar baseline patient characteristics on admission, LVEDV identified on CMRI 2 weeks later was significantly smaller, while diastolic SR in the non-infarcted (rather than infarcted) area was significantly greater in the Impella group than in the non-Impella group, despite 
no difference in the infarct size; and 2) there was no difference in the length of hospital stay between the two groups. All patients were discharged on foot; 3 patients in the non-Impella group required readmission due to heart failure, while no patients experienced heart failure in the Impella group.

\section{Effect of Impella on the prevention of LV remodeling}

It was widely known that shortening the DTBT enables reduction of infarct size; however, use of an Impella has not yet been demonstrated to reduce infarct size in humans. ${ }^{13}$ When assessed via CMRI, our study showed no reduction in infarct size in the Impella group when compared with the non-Impella group. Despite the similar transthoracic echocardiographic LVEF, LVEDV, and LVESV on admission between the two groups, CMRI 2 weeks later revealed that LVEDV was predominantly reduced, while LVESV and LV mass tended to be smaller (despite no statistical significance) by LV unloading in the Impella group. This study also details LV function by systolic and diastolic SRs, segmented by global LV, infarcted, and non-infarcted areas. Myocardial strain (defined as the change in length of an object relative to its original length) is a sensitive measure of contractility, which can be calculated in a variety of coordinate systems at both the segmental and global level; it is typically determined in the three axes of myocardial contraction (circumferential, longitudinal, and radial). ${ }^{14}$

SR measures the change in strain for a given vector as a function of time. Global myocardial circumferential systolic strain and diastolic SR were reported to be objective, sensitive markers of myocardial systolic and diastolic function. ${ }^{15}$ Another report demonstrated that both longitudinal and circumferential systolic SR were independent predictors of outcomes after Ml, whereas only circumferential systolic SR was predictive of remodeling. The data suggested that preserved circumferential function might serve to restrain ventricular enlargement after MI. ${ }^{16}$ Despite no difference in systolic SR during the acute phase, our data showed that magnitudes of diastolic SR in overall areas were significantly greater in the Impella group, and pronounced in the non-infarcted areas in particular. Our findings, together with recent reports, suggest that Impella reduced diastolic dysfunction in the noninfarcted, rather than infarcted area by unloading the entire LV. Bulluck et al. demonstrated increased extracellular volume fraction of the remote myocardium acutely and at $5 \pm 2$ months after STEMI in patients who developed adverse LV remodeling (defined as $\geq 20 \%$ increase in LV end diastolic volume). ${ }^{17}$ Moreover, remote zone non-contrast T1-mapping provided independent and incremental prognostic information above the clinical risk factors and traditional CMRI outcome markers in STEMI patients treated by primary PCl. ${ }^{18}$ The mechanism for improving the non-infarct area by Impella is incompletely unknown; however, recent findings indicate that post-MI LV remodeling is a multifactorial process that may involve excessive inflammation and/or fibrosis of the remote (non-infarct) myocardium; progression may be protected by the Impella.

\section{Outcomes after Impella placement}

Diastolic SR in all LV sites improved, and LV chamber size reduced after using Impella, despite no difference in NT-proBNP, LGE, and length of hospital stay at discharge between the patients with and 
without Impella. While it is known that an increase in LVEDV is an important risk for heart failure, ${ }^{17,18}$ End-systolic volume and LV mass are also been reportedly predictive factors for heart accidents. ${ }^{19,20}$ Ersboll et al. showed that diastolic SR was a sensitive marker of diastolic dysfunction that might occur early in STEMI, independent of systolic dysfunction (strongly associated with adverse outcomes). ${ }^{21}$ Our results, therefore, imply that Impella may provide favorable effects for the prevention of future heart failure.

Recent randomized control trials or observation studies have been shown controversial results regarding the prognostic effect of Impella. The small IMPRESS in Severe SHOCK trial $(n=48)$ did not show any beneficial effects of the Impella CP, compared with IABP. ${ }^{9}$ A recent matched-pair analysis $(237$ matched pairs) comparing Impella- and IABP-treated CS patients showed similar 30-day mortality in both groups. ${ }^{22}$ The lactate level was lower and baseline LVEF assessed by transthoracic echocardiography was higher in our Impella patients than in previous reports, suggesting a better condition in our study patients, possibly due to the early DTBT time in this study. Furthermore, Impella was placed before PCI in all study patients. Early unloading of Impella in our patients may provide protective effects on cardiac dysfunction, leading to subsequent clinical outcomes. This is implied by our results; 3 patients $(19 \%)$ in the non-Impella group suffered rehospitalization due to heart failure, while none in the Impella group suffered any heart failure events. The STEMI-DTU Trial (NCT03947619) is currently ongoing in the United States; the purpose of this study is to evaluate whether using the Impella CP System for 30 minutes prior to catheterization can reduce damage to the heart caused by heart attack, compared with the current standard of care. This trial would answer our questions whether early unloading of Impella improve clinical outcomes in MI with cardiogenic shock.

\section{Study limitations}

First, our study is limited by the retrospective design of our analysis. Additionally, our data is the result of a single-center registry providing a limited number of patients with anterior STEMI. Despite the similar patient characteristics, IABP is generally placed in MI patients without severe risks; healthier bias in the non-Impella group might persist. Nonetheless, LV diastolic function and smaller LV chambers were observed in the Impella group. However, LV ejection fraction assessed by transthoracic echocardiography was lower at baseline; improvement of LV function might have been reasonable. The follow-up period was too short to obtain a statistical difference in clinical outcomes between the Impella and non-Impella groups. Further larger studies in a long period are required to identify the prognostic effect of Impella.

\section{Conclusions}

Early implantation of the Impella before $\mathrm{PCl}$ reduced enlargement of LVEDV and improved diastolic function (diastolic SR) assessed by CMRI. This study provides clinical insight into understanding the usefulness of the Impella for the cardioprotective effects preventing LV remodeling.

\section{Abbreviations}


AMI, acute myocardial infarction; BMI, body mass index; CMRI, cardiac magnetic resonance image; DTBT, door-to-balloon time; eGFR, estimated glomerular filtration rate; IABP, intra-aortic balloon pumping; LAD, left anterior descending; LGE, late gadolinium enhancement; LV, left ventricular; LVEDV, left ventricular end-diastole volume; LVEF, left ventricular ejection fraction; LVESV, left ventricular end-systolic volume; MI, myocardial infarction; SSFP, standard steady-state free precession; NT-proBNP, N-terminal pro-brain natriuretic peptide; $\mathrm{Cl}$, percutaneous coronary intervention; SR, systolic strain rate; STEMI, ST-elevation myocardial infarction; VA-ECMO, veno-arterial extracorporeal membrane oxygenation

\section{Declarations}

Ethics approval and consent to participate. All procedures were performed according to the ethical standards of the institutional research committee, as well as the 1964 Helsinki Declaration and its later amendments or comparable ethical standards. The study and its protocols were approved by the Institutional Review Board of Nihon University Itabashi Hospital (RK-200714-10)..

Consent for publication: Not applicable..

Availability of data and materials: The datasets used and/or analysed during the current study are available from the corresponding author on reasonable request.

Competing interests. Dr. Okumura belongs to the endowed departments of Boston Scientific Japan, Abbott Medical Japan, Japan Lifeline, Medtronic Japan, and Nihon Kohden.

Funding. Not applicable.

Authors' contributions: AY and HF had analyzed CMR data. All authors read and approved the final manuscript.

Acknowledgments: The author thanks Ms. Wendy Alexander-Adams and Mr. John Martin for their encouragement and assistance in the preparation of this commentary in English. We also thank Mr. Akimasa Yamada and Hidehiro Fujito for assistance with the analysis of CMRI.

\section{References}

[1] Takii T, Yasuda S, Takahashi J, Ito K, Shiba N, Shirato K, et al. Trends in acute myocardial infarction incidence and mortality over 30 years in Japan: report from the Miyagi-AMI Registry Study. Circ J. 2010;74:93-100.

[2] Menees DS, Peterson ED, Wang Y, Curtis JP, Messenger JC, Rumsfeld JS, et al. Door-to-balloon time and mortality among patients undergoing primary PCl. N Engl J Med. 2013;369:901-9.

[3] Benjamin EJ, Blaha MJ, Chiuve SE, Cushman M, Das SR, Deo R, et al. Heart disease and stroke Statistics-2017 update: A report from the American Heart Association. Circulation. 2017;135:e146-603. 
[4] Scheidt S, Wilner G, Mueller H, Summers D, Lesch M, Wolff G, et al. Intra-aortic balloon counterpulsation in cardiogenic shock. Report of a co-operative clinical trial. N Engl J Med. 1973;288:97984.

[5] Thiele H, Zeymer U, Neumann FJ, Ferenc M, Olbrich HG, Hausleiter J, et al. Intraaortic balloon support for myocardial infarction with cardiogenic shock. N Engl J Med. 2012;367:1287-96.

[6] Burkhoff D, Sayer G, Doshi D, Uriel N. Hemodynamics of mechanical circulatory support. J Am Coll Cardiol. 2015;66:2663-74.

[7] Remmelink M, Sjauw KD, Henriques JP, de Winter RJ, Koch KT, et al. Effects of left ventricular unloading by Impella recover LP2.5 on coronary hemodynamics. Catheter Cardiovasc Interv. 2007;70:5327.

[8] Remmelink M, Sjauw KD, Henriques JP, de Winter RJ, Vis MM, Koch KT, et al. Effects of mechanical left ventricular unloading by Impella on left ventricular dynamics in high-risk and primary percutaneous coronary intervention patients. Catheter Cardiovasc Interv. 2010;75:187-94.

[9] Ouweneel DM, Eriksen E, Sjauw KD, van Dongen IM, Hirsch A, Packer EJ, et al. Percutaneous mechanical circulatory support versus intra-aortic balloon pump in cardiogenic shock after acute myocardial infarction. J Am Coll Cardiol. 2017;69:278-87.

[10] Amin AP, Spertus JA, Curtis JP, Desai N, Masoudi FA, Bach RG, et al. The evolving landscape of Impella use in the United States among patients undergoing percutaneous coronary intervention with mechanical circulatory support. Circulation. 2020;141:273-84.

[11] Hemradj VV, Karami M, Sjauw KD, Engström AE, Ouweneel DM, de Brabander J, et al. Pre-PCl versus immediate post-PCI Impella initiation in acute myocardial infarction complicated by cardiogenic shock. PLoS One. 2020;15:e0235762.

[12] Nagueh SF, Smiseth OA, Appleton CP, Byrd BF 3rd, Dokainish H, Edvardsen T, et al. Recommendations for the Evaluation of Left Ventricular Diastolic Function by Echocardiography: An Update from the American Society of Echocardiography and the European Association of Cardiovascular Imaging. Eur Heart J Cardiovasc Imaging. 2016;17:1321-60.

[13] Saku K, Kakino T, Arimura T, Sunagawa G, Nishikawa T, Sakamoto T, et al. Left ventricular mechanical unloading by total support of Impella in myocardial infarction reduces infarct size, preserves left ventricular function, and prevents subsequent heart failure in dogs. Circ Heart Fail. 2018;11:e004397.

[14] Nazir SA, Shetye AM, Khan JN, Singh A, Arnold JR, Squire I, et al. Inter-study repeatability of circumferential strain and diastolic strain rate by CMR tagging, feature tracking and tissue tracking in STsegment elevation myocardial infarction. Int J Cardiovasc Imaging. 2020;36:1133-46. 
[15] Khan JN, Wilmot EG, Leggate M, Singh A, Yates T, Nimmo M, et al. Subclinical diastolic dysfunction in young adults with type 2 diabetes mellitus: a multiparametric contrast-enhanced cardiovascular magnetic resonance pilot study assessing potential mechanisms. Eur Heart J Cardiovasc Imaging. 2014;15:1263-9.

[16] Hung CL, Verma A, Uno H, Shin SH, Bourgoun M, Hassanein AH, et al. Longitudinal and circumferential strain rate, left ventricular remodeling, and prognosis after myocardial infarction. J Am Coll Cardiol. 2010;56:1812-22.

[17] Bulluck H, Rosmini S, Abdel-Gadir A, White SK, Bhuva AN, Treibel TA, et al. Automated Extracellular Volume Fraction Mapping Provides Insights Into the Pathophysiology of Left Ventricular Remodeling Post-Reperfused ST-Elevation Myocardial Infarction. J Am Heart Assoc. 2016;5:e003555.

[18] Reinstadler SJ, Stiermaier T, Liebetrau J, Fuernau G, Eitel C, de Waha S, et al. Prognostic significance of remote myocardium alterations assessed by quantitative noncontrast T1 mapping in ST-segment elevation myocardial infarction. JACC Cardiovasc Imaging. 2018;11:411-9.

[19] Verma A, Meris A, Skali H, Ghali JK, Arnold JM, Bourgoun M, et al. Prognostic implications of left ventricular mass and geometry following myocardial infarction: the VALIANT (valsartan in acute myocardial infarction) Echocardiographic Study. JACC Cardiovasc Imaging. 2008;1:582-91.

[20] Solomon SD, Skali H, Anavekar NS, Bourgoun M, Barvik S, Ghali JK, et al. Changes in ventricular size and function in patients treated with valsartan, captopril, or both after myocardial infarction. Circulation. 2005;111:3411-9.

[21] Ersbøll M, Andersen MJ, Valeur N, Mogensen UM, Fakhri Y, Thune JJ, et al. Early diastolic strain rate in relation to systolic and diastolic function and prognosis in acute myocardial infarction: a twodimensional speckle-tracking study. Eur Heart J. 2014;35:648-56.

[22] Schrage B, Ibrahim K, Loehn T, Werner N, Sinning JM, Pappalardo F, et al. Impella support for acute myocardial infarction complicated by cardiogenic shock. Circulation. 2019;139:1249-58.

\section{Figures}




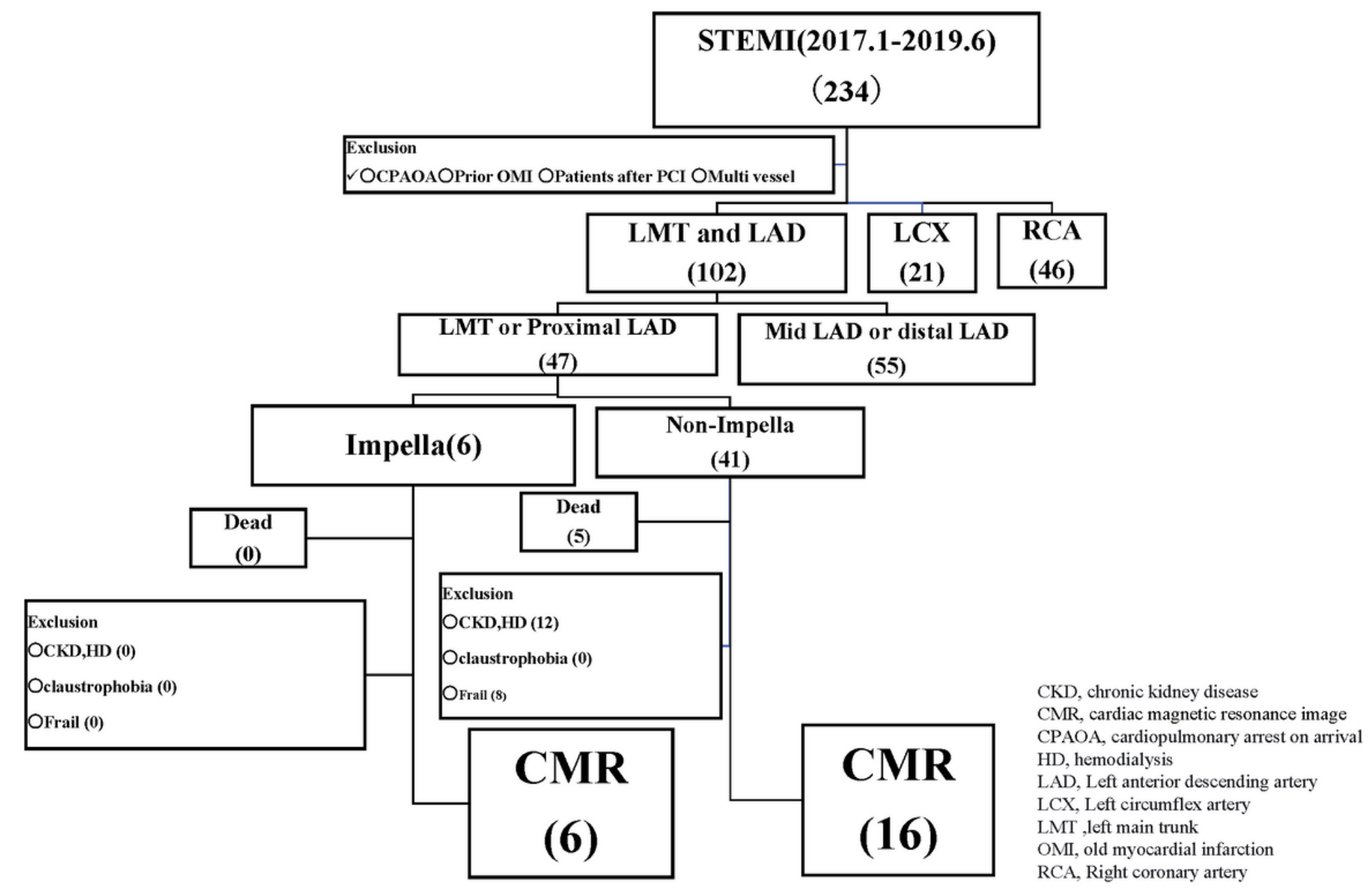

Figure 1

Flow chart of the study entrants 


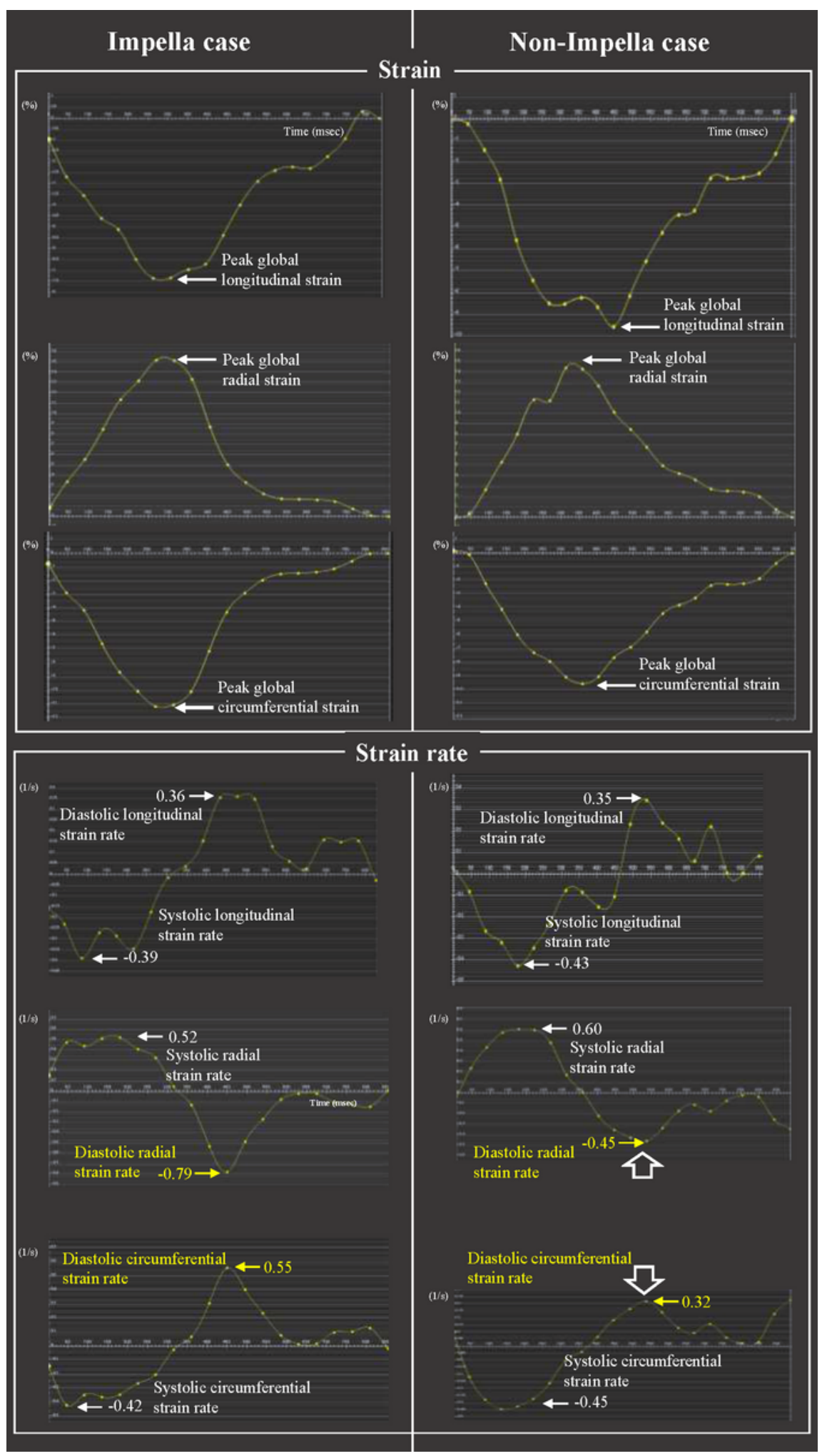

Figure 2

Representative CMRI strains and SR images in the Impella and non-Impella cases. CMRI strains: peak global longitudinal, radial and circumferential strains; SR images: global longitudinal systolic and diastolic SR, radial systolic and diastolic SR, and circumferential systolic and diastolic SRs. Note that despite no difference in global strains, the magnitudes of the diastolic radial and circumferential SRs are 
higher in the Impella group than in the non-Impella group. CMRI, cardiac magnetic resonance imaging; $\mathrm{SR}$, strain rate
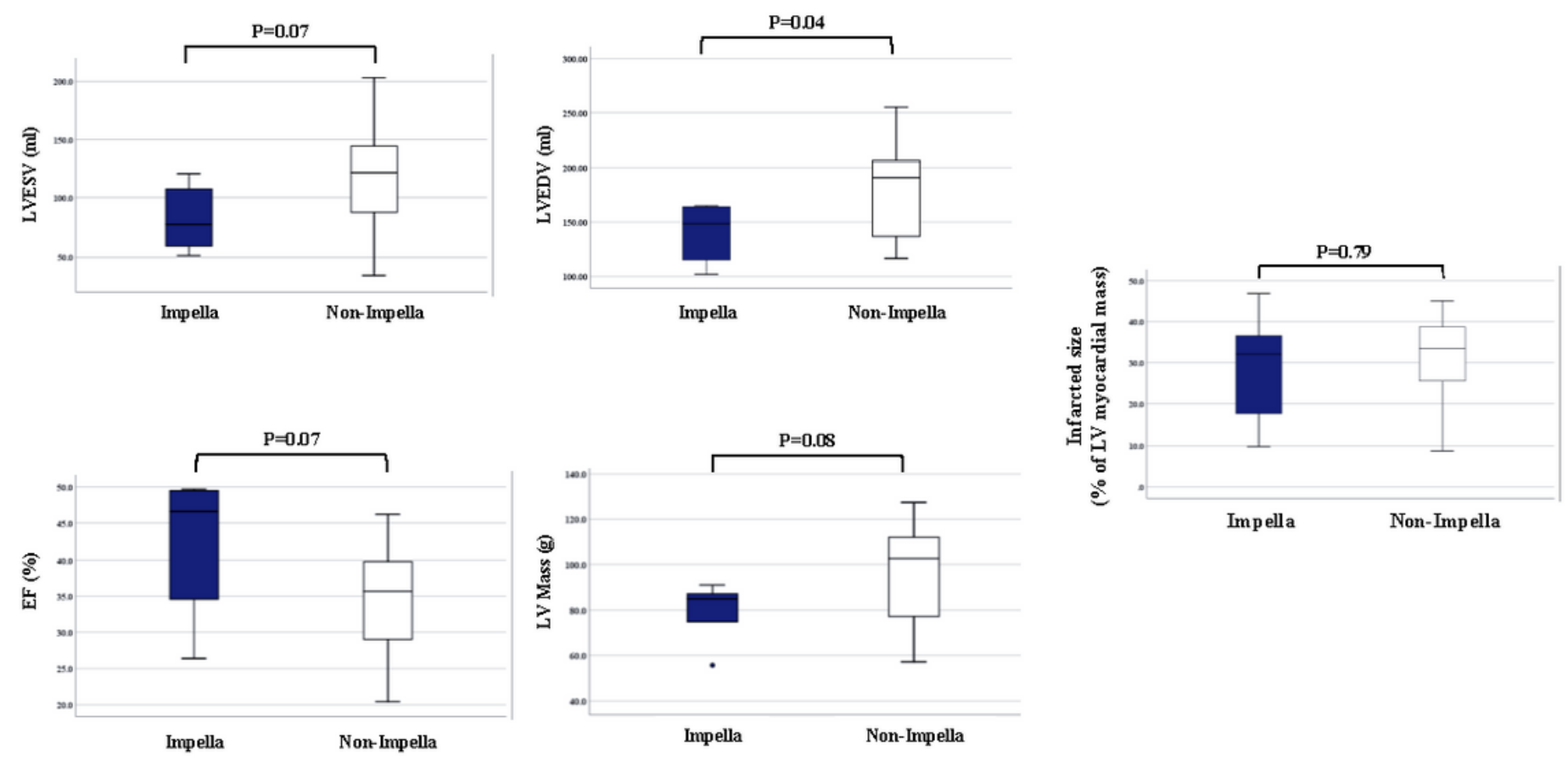

Figure 3

Comparison of LVESV, LVEDV, EF, LV mass, and LGE between the Impella and non-Impella groups EF, ejection fraction; LGE, late gadolinium enhancement; LVEDV, left ventricular end-diastole volume; LVESV, left ventricular end-systolic volume; LV, left ventricular 

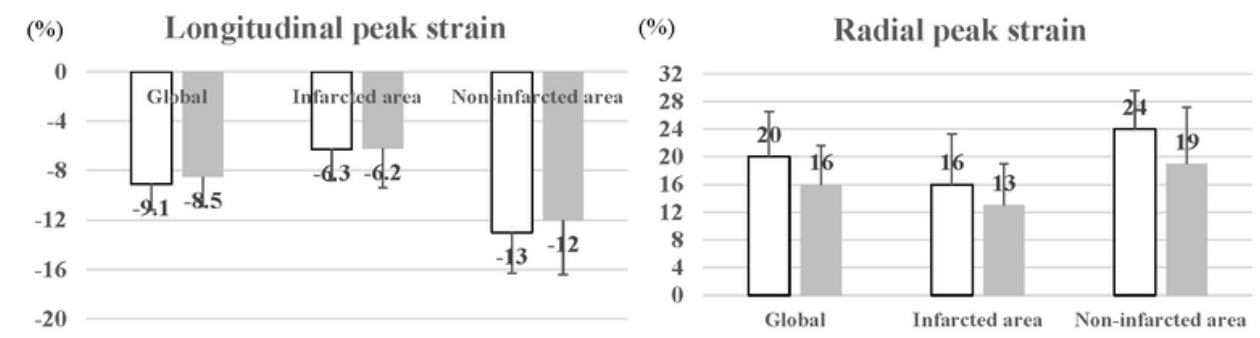

$\left(\mathrm{S}^{-1}\right)$

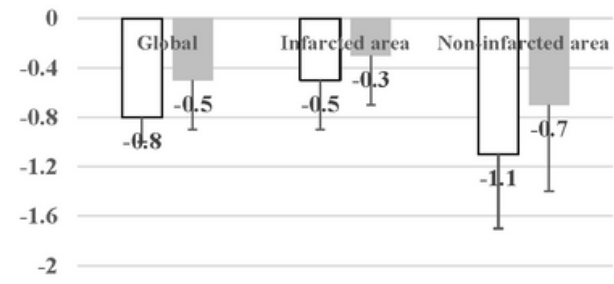

$\left(\mathrm{S}^{-1}\right) \quad$ Longitudinal diastolic strain rate

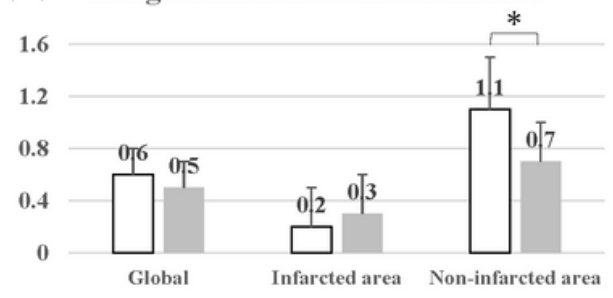

$\left(\mathrm{S}^{-1}\right)$

Radial systolic strain rate

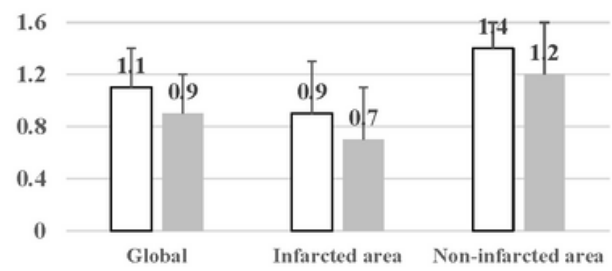

$\left(\mathrm{S}^{-1}\right)$

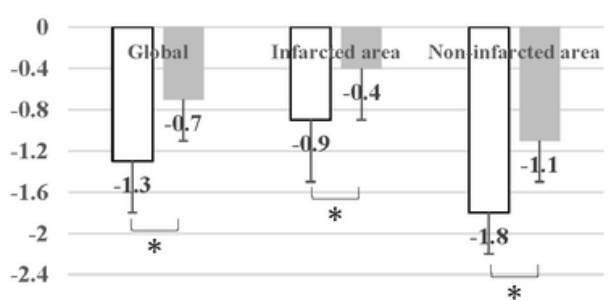

Radial diastolic strain rate
(\%) Circumferential peak strain

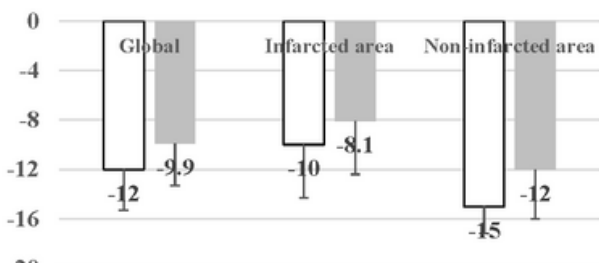

$\left(\mathrm{S}^{-1}\right)$ Circumferential systolic strain rate

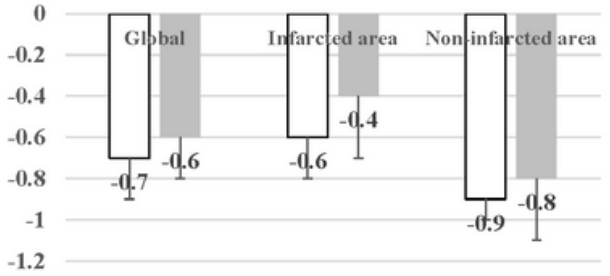

$\left(\mathrm{S}^{-1}\right)$ Circumferential diastolic strain rate

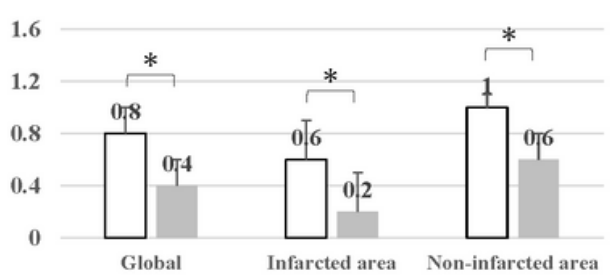

\section{Figure 4}

CMRI strain parameters between the Impella group (white bar) and non-Impella group (grey bar) ${ }^{*}<<0.05$ $\mathrm{CMRI}$, cardiac magnetic resonance imaging 